\title{
Accuracy Validation of Cone-Beam CT Based Registration
}

\author{
Yoshikazu Nakajima ${ }^{1}$, Toshihiko Sasama ${ }^{1}$, Yoshinobu Sato ${ }^{1}$, Takashi Nishii ${ }^{2}$, \\ Nobuhiko Sugano ${ }^{2}$, Takashi Ishikawa ${ }^{4}$, Kazuo Yonenobu ${ }^{2}$, Takahiro Ochi ${ }^{3}$, \\ Shinichi Tamura ${ }^{1}$ \\ ${ }^{1}$ Division of Interdisciplinary Image Analysis \\ 2 Department of Orthopaedic Surgery \\ 3 Division of Computer Integrated Orthopaedic Surgery \\ Osaka University Graduate School of Medicine \\ ${ }^{4}$ Hitachi Medical Corporation \\ nakajima@image.med.osaka-u.ac.jp \\ http://www.image.med.osaka-u.ac.jp/nakajima
}

\begin{abstract}
In this paper, the accuracy of intraoperative registration using cone-beam CT for computer-assisted surgery is preliminarily evaluated. Since cone-beam CT enables whole-surface detection of bone without surgical exposure, highly accurate registration is potentially attainable. In in vitro experiments, much higher accuracy could be attained by acquiring the intraoperative bone surface using cone-beam CT ( 0.56 $\mathrm{mm}$ ) as compared with that of our routinely used protocol employing a 3 -D positional sensor $(1.43 \mathrm{~mm})$.
\end{abstract}

\section{Introduction}

Percutaneous surgical procedures and endoscopic surgeries are being increasingly used because of their minimal invasiveness. However, the surgeon does not have a direct view, or has only a restricted view, of anatomical structures. Hence, computer assistance is useful in these procedures, for which accurate registration between preoperative and intraoperative data without surgical exposure is a key technology.

For registration in computer-assisted bone surgery, the x-ray fluoroscope is suitable for the intraoperative localization of bone without surgical exposure. To improve the accuracy and reliability of registration, combining images from two or more views has been proposed [1]. The cone-beam CT scanner is regarded as an extension of the multiview x-ray fluoroscope, and is compact enough for use in the operating room. While the contrast resolution of the CT value in cone-beam CT is still insufficient for diagnosis involving soft tissues, the spatial resolution is isotropic with respect to all three axes and is thus well-suited to $3-\mathrm{D}$ acquisition. Since bone has inherently high contrast in the CT value, cone-beam CT can be particularly useful for 3-D bone surface scanning. In this paper, we consider the use of cone-beam CT as an intraoperative modality for bone surface detection to attain intraoperative registration with high accuracy without surgical exposure. 


\section{Method}

The accuracy of cone-beam CT based registration (CB-REG) was evaluated by comparing it with 3-D positional sensor based registration (PS-REG) using a pelvis specimen. The experimental methods were as follows.

1. Preoperative surface model generation: A pelvis surface was generated using preoperative $\mathrm{CT}$ images with 3 -mm thickness by using a conventional helical CT scanner.

2. Intraoperative surface point collection: For PS-REG, 30 surface points in the surgical exposure region were sampled using an Optotrak (Northern Digital Inc.) 3-D positional sensor (Fig. П (a)). The number of sample points, 30 , was determined by considering the trade-off between registration accuracy and the time needed for point collection [2]; this number is routinely used for hip-joint surgical navigation in our hospital. (Note that sampling points on the bone surface is not always easy because it is covered in muscle and other tissues.)

For CB-REG, a pelvis surface was first generated from cone-beam CT (Hitachi Medical Corp.) images by using the same process as that employed for surface generation from helical CT images. After removing the surfaces of artificial markers (ceramic balls, described below) for accuracy evaluation, intraoperative points on the pelvis surface were collected automatically and randomly. Registration was performed using three protocols. In the first (CB-REG1), 30 surface points were collected in the surgical exposure region on the pelvis surface generated from the cone-beam CT images (Fig. [1(b)) to examine the difference in accuracy between point collection by Optotrak and cone-beam CT. In the other two protocols, which were designed to examine differences arising from the extent of the sample region, 1000 surface points were collected in the surgical exposure region (CB-REG2) or over the whole surface (CB-REG3. Fig. 1(c)).

3. Registration: By using the iterative closest point algorithm, registration between the preoperative pelvis surface and the intraoperative sample points was performed.

4. Accuracy evaluation: The target registration error (TRE) [3] at the acetabular center was calculated by using the gold-standard of registration, which had been estimated from four micron-accurate ceramic balls fixed to the pelvis surface. The average and standard deviation of the TRE were evaluated using 20 sets of sample points for each protocol.

\section{Results and Discussion}

As shown in Table 1 by using the same number of sample points (30) and the same sampling area (surgical exposure), the average TRE was improved from $1.43 \mathrm{~mm}$ (PS-REG) using Optotrak to $0.80 \mathrm{~mm}$ (CB-REG1) using cone-beam CT. By increasing the number of sample points from 30 to 1000, the standard deviation (SD) of the TRE was improved from $0.73 \mathrm{~mm}$ (CB-REG1) to $0.13 \mathrm{~mm}$ 
(CB-REG2), while the average remained almost the same. Further, by expanding the sampling area to the whole surface, the average TRE was improved to $0.56 \mathrm{~mm}$ (CB-REG3). In summary, the findings showed that registration with much higher accuracy is potentially attainable by acquiring the intraoperative bone surface using cone-beam CT as compared with our current routinely used protocol (PS-REG).

We consider that $0.56 \mathrm{~mm}$, which was derived from in vitro experiments, can be regarded as the upper limit of accuracy attainable using cone-beam CT. In future work, we aim to study how the degree of accuracy using in vivo images can be brought close to this upper limit. Furthermore, since cone-beam CT based registration entails radioactive exposure, the overall advantages and disadvantages to the patient will need be sufficiently considered in clinical application.

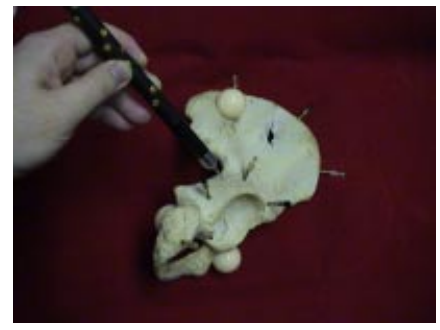

(a) Sampling using 3-D positional sensor

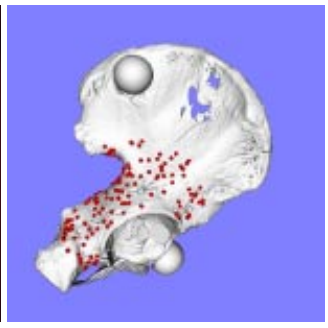

(b) Sampling in the surgical exposure region

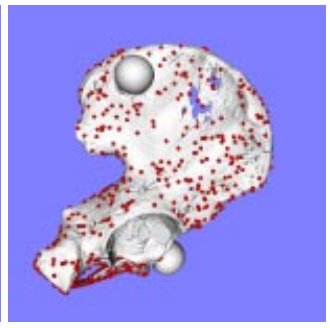

(c) Sampling of the whole surface

Fig. 1. Intraoperative point sampling on the pelvis surface

Table 1. Results of accuracy evaluation

\begin{tabular}{|c|c|c|c|c|}
\hline & PS-REG & \multicolumn{3}{|c|}{ 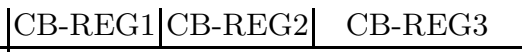 } \\
\hline Sample method & $\begin{array}{l}\text { Point collection } \\
\text { by using } 3 \text {-D po- } \\
\text { sitional sensor }\end{array}$ & \multicolumn{3}{|c|}{$\begin{array}{l}\text { Point collection on the bone surface } \\
\text { generated from cone-beam CT im- } \\
\text { ages }\end{array}$} \\
\hline Sample number & \multicolumn{3}{|c|}{\begin{tabular}{|l|l|}
30 & 1 \\
\end{tabular}} & 1000 \\
\hline Sample region & \multicolumn{3}{|c|}{ Surgical exposure region } & Whole surface \\
\hline Average TRE (in & 1.43 & 0.80 & 0.82 & 0.56 \\
\hline Standard deviation (in $\mathrm{mm}$ ) & 2.06 & 0.73 & 0.13 & 0.24 \\
\hline
\end{tabular}

\section{References}

1. S. Lavalée, R. Szelski: Recovering the Position and Orientation of Free-Form Objects from Image Contours Using 3D Distance Maps, IEEE Trans. on PAMI, 17 (4), 378-390 (1995).

2. T. Sasama, Y. Sato, N. Sugano, el al.: Accuracy Evaluation in Computer Assisted Hip Surgery, Computer Assisted Radiology and Surgery (CARS'99), Paris, 772-776 (1999).

3. C.R. Maurer, J.M. Fitzpatrick, M.Y. Wang, et al.: Registration of Head Volume Images Using Implantable Fiducial Markers, IEEE Trans. on Medical Imaging, 16 (4), 447-462 (1997). 